\title{
Development and validation of the Avoidance of Respiratory Viral Infection Scale (ARVIS)
}

\author{
Toby Wise ${ }^{1,2,3}$, Giorgia Michelini ${ }^{4}$, Tomislav Zbozinek ${ }^{1}$, Cindy C Hagan ${ }^{1}$ \& Dean Mobbs ${ }^{1,5}$ \\ ${ }^{1}$ Division of Humanities and Social Sciences, California Institute of Technology, Pasadena, CA \\ ${ }^{2}$ Max Planck UCL Centre for Computational Psychiatry and Ageing Research, University College London, \\ London, UK \\ ${ }^{3}$ Wellcome Centre for Human Neuroimaging, University College London, London, UK \\ ${ }^{4}$ Semel Institute for Neuroscience and Human Behavior, University of California Los Angeles, Los Angeles, \\ CA \\ ${ }^{5}$ Computational Neural Systems Program, California Institute of Technology, Pasadena, CA
}

\begin{abstract}
During respiratory viral epidemics and pandemics such as the COVD-19 pandemic, everyday behaviors such as leaving the house and touching frequently-used surfaces become the subject of fear and avoidance due to their potential for contagion. Despite the potential mental health related impact of this psychological phenomenon, there are currently no measures available that are designed to evaluate the extent to which individuals become fearful of these everyday situations during a respiratory viral pandemic. Here, we developed and assessed the psychometric properties of the Avoidance of Respiratory Viral Infection Scale (ARVIS) questionnaire across two samples recruited online $(n s=243,341)$ recruited during the COVID-19 pandemic. Our results indicate that the ARVIS is best described by a single factor and shows a high reliability (Chronbach's alpha $>$.88). It showed convergent associations with state anxiety and low discriminant associations with general trait anxiety and depression. Moreover, the ARVIS was highly associated with the extent to which subjects reported engaging in handwashing and social distancing, demonstrating criterion validity. This new scale provides a validated measure for assessing virus-related fear that can facilitate research into the psychological effects of respiratory virus pandemics.
\end{abstract}




\section{Introduction}

Respiratory virus epidemics and pandemics occur frequently (Madhav et al., 2017) and, depending on their spread and severity, can have serious consequences both locally and across the globe (Lai et al., 2020; Oxford, 2000). As these viruses tend to spread through aerosols and droplets shed by infected individuals (Cowling et al., 2013; Leung et al., 2020; Tellier, 2009), the most likely sources of infection are close contact with others and touching surfaces that are contaminated with viral matter (Killingley \& Nguyen-Van-Tam, 2013). Because of this, the most commonly recommended protective measure that an individual can take to reduce the risk of infection are social distancing, through avoiding close contact with others, and disinfecting potentially infected surfaces, such as door handles or one's own hands (World Health Organisation, 2006). However, the implied association between everyday behaviors such as close physical interaction with others and touching frequently-used surfaces has the potential to lead to fear of actions that were previously considered harmless.

This fear of everyday situations is of interest for distinct but interlinked reasons. First, from the perspective of public health, a realistic, accurate, degree of adaptive fear in response to situations associated with virus transmission may be helpful in motiving people to engage in protective behaviors that are necessary to stem the spread of a pandemic or epidemic. It has already been shown that perceived threat and general pandemic-related fear are associated with increased engagement in protective behaviors (Bish \& Michie, 2010; Bults et al., 2011; Harper et al., 2020; Wise et al., 2020), however it remains unknown whether fears of everyday situations specifically play a role in motivating engagement. Second, from a clinical psychology perspective, while this fear is adaptive in the acute phase of a pandemic, it may persist after the immediate threat has dissipated (Dutheil et al., 2020; Perrin et al., 2009; Xu et al., 2011), as is possible following traumatic experiences more generally (Breslau, 2009; Bromet et al., 2016). As a result, what is an adaptive fear response during the acute phase may become a pathological fear in time with a negative impact on an individual's ability to function in everyday life. Being able to measure these fears accurately could aid in the assessment and treatment of pathological fear in the absence of an acute pandemic. Third, from the perspective of more basic behavioral science, the acquired fear of previously safe stimuli represents a naturalistic form of context-dependent fear conditioning (Maren et al., 2013). Therefore, understanding how pandemic-related fear of everyday situations is learned and extinguished has the potential to provide a more ecologically valid understanding of fear learning.

Despite the importance of pandemic-acquired fear of everyday situations to a range of disciplines, there are currently no validated measures that can be used to assess the extent to which individuals demonstrate respiratory virus-related fear of everyday situations. This is likely due, in part, to the challenges inherent in developing a measure that assesses a state variable that only manifests strongly in pandemics. While other scales have been developed to assess pandemic-related fear during the COVID-19 pandemic (Arpaci et al., 2020; Conway et al., 2020; Kachanoff et al., 2020) and other pandemics (Arrindell et al., 1989; Harrell \& Wright, 1998; Wheaton et al., 2012), these typically focus on perceived threat to the individual or society and often include items that are specific to the pandemic under study, limiting their use in other respiratory viral pandemics and epidemics.

We developed and validated the Avoidance of Respiratory Viral Infection Scale (ARVIS) questionnaire during the COVID-19 pandemic, with the aim of producing a robust measure of the extent to which individuals feared everyday situations. Although we tested this scale during a specific pandemic, we designed the scale to be general in its phrasing and applicable to any situation in which individuals may be exposed to a respiratory virus. We evaluated the psychometric properties of the measure across two samples and assessed convergent, discriminant, and criterion validity. Finally, we evaluated test-retest reliability in a subset of the first sample who completed the measure after one week. 


\section{Methods}

\section{Samples}

We collected two samples of subjects online using Prolific (Palan \& Schitter, 2018) (Study $1 \mathrm{n}=243$, Study $2 n=341$ ) on $5 / 18 / 2020$ and $5 / 21 / 2020$ respectively. These sample sizes are adequate for exploratory factor analysis (EFA) and confirmatory factor analysis (CFA), assuming simple models and reasonably high factor loadings, respectively (de Winter et al., 2009; Wolf et al., 2013). Subjects were selected based on being located in the United States and having over a 95\% approval rating on Prolific. Additionally, in Study 3, we collected data from 202 subjects who completed Study 1 one week later to evaluate test-retest reliability. These subjects took part as part of a larger longitudinal study into psychological responses to the COVID19 pandemic (Wise et al., 2020). The study was approved by the Institutional Review Board at the California Institute of Technology. All subjects provided informed consent prior to participation.

Procedure and analyses

In Study 1, we first administered a battery of 26 candidate questions designed to measure fear and avoidance of ordinary, everyday behaviors such as touching door handles and coming into close contact with other people. Various sources were used to generate items, including news articles describing fears and anxiety in relation to the pandemic, prior results from studies during the pandemic (Conway et al., 2020; Kachanoff et al., 2020; Wise et al., 2020), and measures of virus-related fears in prior pandemics. Our aim was to assess emotional reactions to situations and behaviors that would ordinarily not elicit a negative emotional reaction in the majority of individuals, outside the context of a respiratory virus pandemic. Items broadly referred to four situations or behaviors: coming into close contact with others (e.g. coming within $6 \mathrm{ft}$ of a stranger), being in situations where large numbers of other people are likely to be encountered (e.g. attending a concert), touching potentially infected surfaces (e.g. touching door handles), and witnessing signs of infection (e.g. hearing. Each item was rated on a five-point Likert scale with the following options: (strongly disagree, moderately disagree, neither agree nor disagree, moderately agree, strongly agree). Items were designed to be simple to understand and include situations that most people would encounter frequently.

\section{Data quality}

In order to ensure high data quality, we included a single attention check question, where subjects were asked to select "strongly agree" as their response. Subjects who did not pass this check were excluded from analysis.

\section{Item selection and reliability}

We initially examined response distributions to identify those with skewed responses or low endorsement rates, rejecting items based on visual inspection of response distribution histograms. We also assessed inter-item correlations to identify items that showed low correlations with others, removing any items that had an average Pearson correlation below.4. We then examined internal consistency using both Cronbach's alpha and omega (Dunn et al., 2014), using a cut-off of .80 to indicate high reliability.

\section{Exploratory factor analysis}

Prior to performing EFA, we used Bartlett's test of sphericity (Dziuban \& Shirkey, 1974) to determine whether the data were amenable to factor analysis, using $p<.05$ as a cutoff. We then performed maximum likelihood EFA using minimal residual extraction, as implemented in the Psych package in $\mathrm{R}$ (version 3.6.3). We expected the measure to either have a unidimensional structure or measure highly correlated constructs, and so used an oblique rotation. 
To determine the appropriate number of factors, we used three primary metrics. First, we performed parallel analysis (Hayton et al., 2004), which involved comparing the solution obtained from the true data to solutions obtained through running the EFA procedure on simulated data. We then assessed the Root Mean Square Error Approximation (RMSEA), where values $<.10$ represent good fit, and values $<.08$ represent excellent fit (Browne \& Cudeck, 1992). Finally, we used the Bayesian Information Criterion (BIC), which provides an index of comparative model fit accounting for model complexity.

Following EFA, we removed items that either had no strong loadings (below .3) or had high cross loadings (having a difference lower than 4 between loadings onto different factors). Following this, we re-evaluated reliability and factor model fit to ensure that removal of certain items did not affect the structure. Finally, in order to reduce the length of the measure we evaluated model fit and reliability when retaining only the strongest loading items.

Confirmatory factor analysis

Following EFA, we sought to confirm the structure of the measure in our second, independent sample (Study 2) using CFA implemented in Lavaan (Rosseel, 2012). We evaluated fit using multiple fit indices, including RMSEA, $X^{2}$, Comparative Fit Index (CFI), standardized root mean square residual (SRMR), BIC, and Akaike Information Criterion (AIC). We followed this by inspecting modification indices to identify potential areas for improvement in the factor model and adjusting the model specification as required (Perry et al., 2015).

Convergent and discriminant validity

We sought to verify the convergent and discriminant validity of the measure using a range of measures completed by a subset of our second sample $(n=272)$. These measures were as follows.

Overall Anxiety Severity and Impairment Scale (OASIS), state measure (Campbell-Sills et al., 2009). The OASIS is a five-item scale that assesses a range of symptoms of state anxiety. As we expected our measure to be a state measure, fluctuating in line with pandemic-related risk, our primary convergent measure was state anxiety. Since virus-related fear is likely to evolve along with state anxiety, we expected the ARVIS scale to show a high correlation with the OASIS.

Short Health Anxiety Inventory (SHAl) (Salkovskis et al., 2002). The 14-item SHAl assesses general healthrelated anxiety, not specifically linked to any particular form of health problem. While not a state measure, and not specifically related to pandemics or respiratory viruses, we expected a small to moderate correlation with general health anxiety. Due to a data recording error, responses were only recorded for the first 13 items.

State Trait Inventory of Cognitive and Somatic Anxiety (STICSA), trait scale (Grös et al., 2007). The 21-item STICSA assesses cognitive and somatic trait anxiety, and has been shown to be a more valid measure than other measures such as the State-Trait Anxiety Inventory (Spielberger, 1983). We expected our measure to be weakly correlated with trait anxiety as, while trait anxiety may predispose individuals to a greater degree of virus-related anxiety, virus-related anxiety should be far more state and environment dependent, and therefore relatively unrelated to trait anxiety.

Beck Depression Inventory (BDI) (Beck, 1978). The BDI is an extensively used and well-validated 21-item measure of depressive symptoms. We elected to use this measure to assess discriminative validity as we did not expect virus-related fears to be related to depressive symptoms. We omitted the item asking about suicidal ideation as we were unable to fully assess risk in an online sample.

Social Interaction Anxiety Scale (SIAS) and Social Phobia Scale (SPS), short forms (Fergus et al., 2014). These five-item measures assessing social anxiety. Many of the items in our measure related to avoidance 
of social interaction, and so it was important to demonstrate that it is not simply measuring a general level of social anxiety (i.e. evidence of discriminant validity).

COVID threat scale (Kachanoff et al., 2020). This scale is designed to measure perceived threat from the COVID-19 pandemic. The measure has two subscales, the first of which is termed "symbolic threat" and represents threats to American values such as democracy and freedom, while the second is termed "realistic threat" and represents threats to health and financial safety. We expected the ARVIS to correlate positively with the realistic threat subscale, and to correlate minimally with the symbolic threat subscale.

These measures were either completed in the same session (SHAI, SIAS, SPS) or in a separate session two weeks prior to completing the ARVIS (BDI, STICSA). We assessed convergent and discriminant validity by examining correlations between their scores and scores on the ARVIS. We then tested for significant differences in the correlations between the ARVIS and our measures of convergent validity, and correlations between the ARVIS and those used to assess discriminant validity (Hittner et al., 2003). Our aim was to assess convergent and discriminant validity based on the difference in correlations with related and unrelated measures, rather than simply examining raw correlation coefficients, to provide evidence that our measure predicts constructs that are expected to be related significantly better than those expected to be unrelated (Clark \& Watson, 2019).

\section{Criterion validity}

We assessed criterion validity by examining relationships between the ARVIS and reported behavior expected to be correlated with virus-related fear of everyday situations. For this purpose, we examined correlations between ARVIS scores and four items designed to measure self-reported engagement in protective behaviors during the pandemic (measured in the same session), namely handwashing, avoidance of social interactions, and staying home. As the behavior items were highly negatively skewed, we report Spearman correlation coefficients. These items were phrased as follows: "The virus has caused me to avoid in-person social interactions (e.g., friends, family, co-workers, strangers)", "I am washing my hands more than I would normally", and "I am staying home more than I normally would". These items were rated on a visual analogue scale ranging from "strongly disagree" to "strongly agree".

Test-retest reliability

Finally, in Study 3, we evaluated test-retest reliability over a one-week period in a subset of subjects who completed Study 1 . We assessed reliability using the Pearson $r$ correlation between scores at the two time points, where values closer to 1 indicate higher consistency between time points. Additionally, we used twoway intraclass correlation coefficients (ICC). Specifically, we used ICC(A, 1), as described by (McGraw \& Wong, 1996) to assess absolute agreement across time points, and ICC (C, 1) to assess consistency of scores across time points. The ICC ranges between 0 and 1, with values below . 5 indicating poor reliability, .5 to 
.75 indicating moderate reliability, .75-.9 indicating good reliability, and values over .9 indicating excellent reliability (Koo \& Li, 2016).
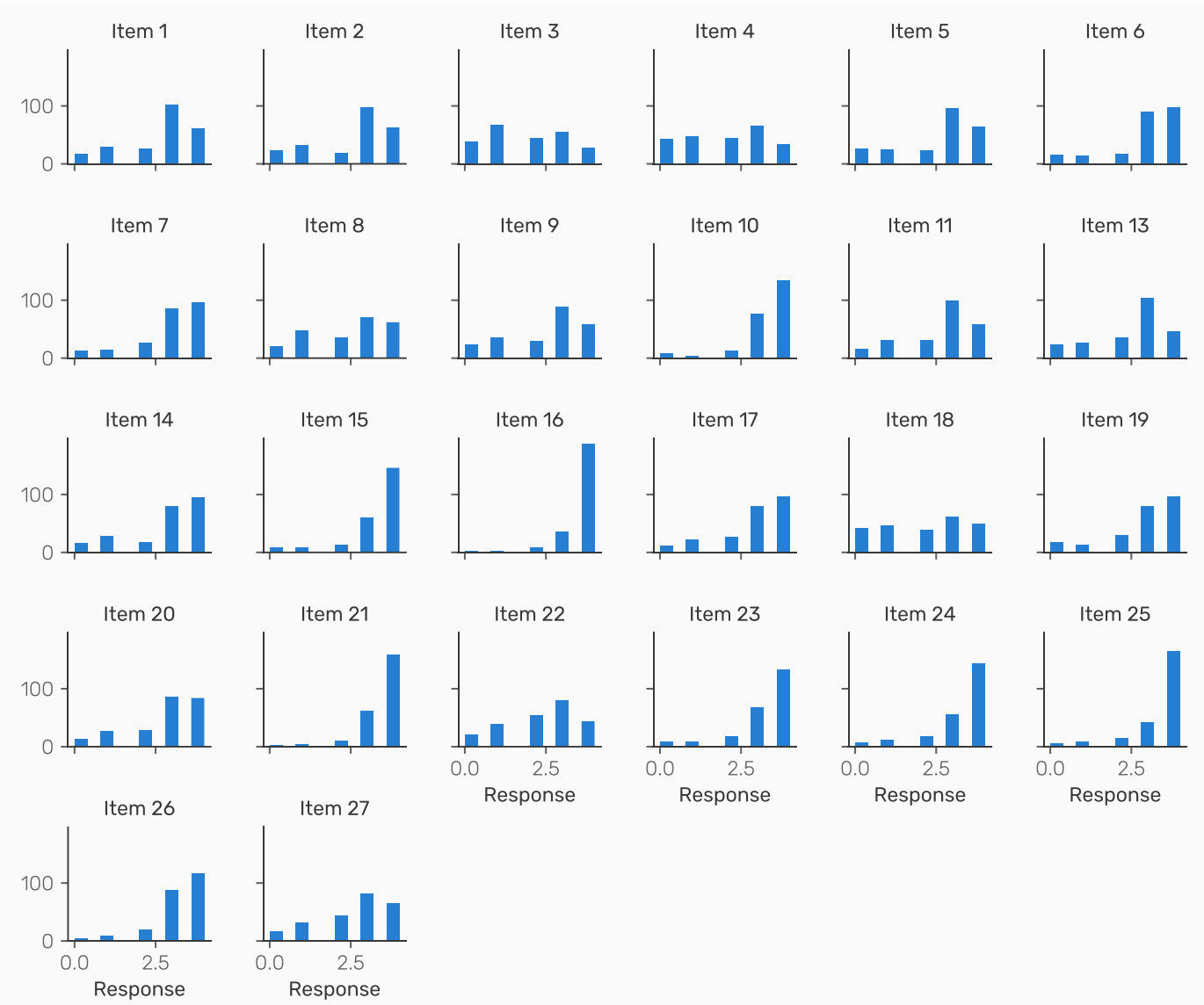

Figure 1. Histograms showing response distributions for each item in Study 1. Item descriptions are provided in Table S1. Item 12 was an attention check question and is not reported here.

\section{Results}

\section{Study 1}

Response distributions and item correlations

Six subjects who failed the attention check were excluded. Examination of response distributions revealed a number of items $(10,15,16,21,23,24,25$, and 26) with highly negatively skewed response distributions (Figure 1), with very few subjects responding 'strongly disagree'. These items mostly focused on attending events with large numbers of people. As a result, these items were removed prior to further analysis.

Inter-item correlations identified three items $(8,18,19)$ that demonstrated low correlations with other items (shown in Figure 2), which were removed. 


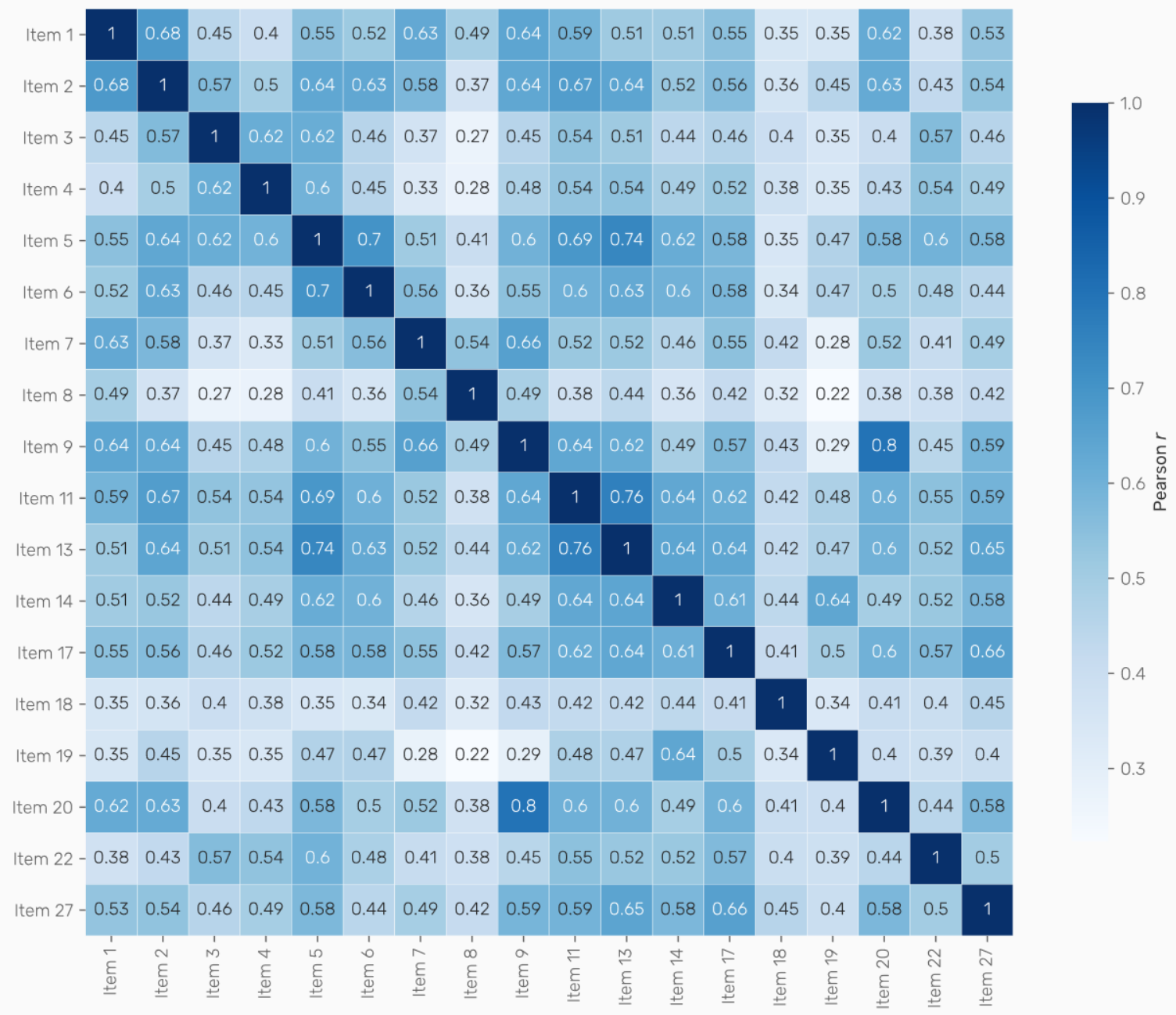

Figure 2. Inter-item Pearson's correlations

Reliability

The measure showed Cronbach's alpha $=0.95$ and omega $=0.96$. Both these values demonstrate high reliability. 


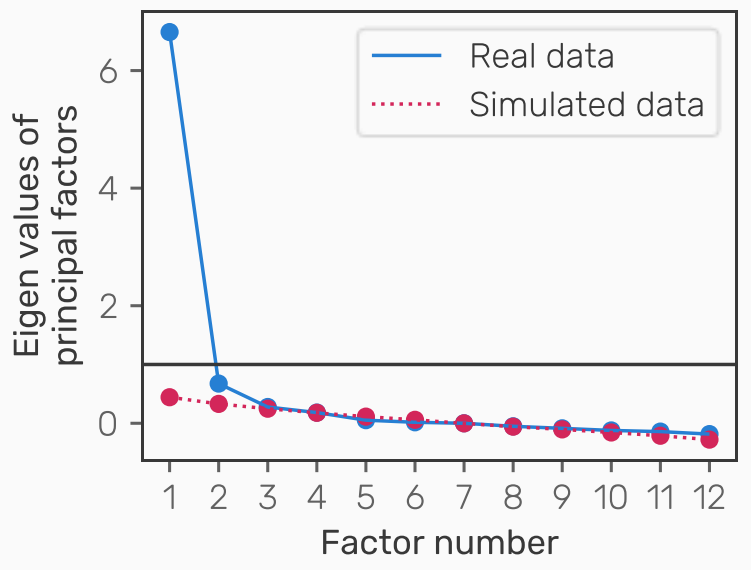

Figure 3. Scree plot results of parallel analysis used to identify the appropriate number of factors in the exploratory factor analysis. The horizontal line represents an eigenvalue of 1. Parallel analysis selects factors based on having higher eigenvalues than those of simulated data.

\section{Exploratory factor analysis}

Bartlett's test of sphericity was significant $\left(X^{2}(105)=2569.44, p<.001\right)$, and so we proceeded to run exploratory factor analysis. Parallel analysis suggested that the existence of two factors. Factor loadings for this 2-factor model are shown in Table 1. This was supported by the BIC scores, which indicated that the two-factor model $(\mathrm{BIC}=-178.13)$ had better fit than a one factor model $(\mathrm{BIC}=-103.79)$ and a three-factor model $(\mathrm{BIC}=-158.22)$. The RMSEA indicated that this model provided a good, although not excellent, fit to the data, with a value of 0.094 . The first factor accounted for $65 \%$ of the variance, while the second accounted for $35 \%$. However, these factors were highly correlated at $r=.77$. Items loading onto these factors broadly represented fear of social interactions and fear of infection, respectively.

We next inspected item loadings to identify items that were either loading weakly or loading nondiscriminatively between factors (Table 1 ). This led us to remove a further three items $(2,17,27)$. We subsequently checked the reliability of the measure with these items removed, which was still excellent (alpha $=.94$, omega $=.95$ ). We also reran the factor analysis, again confirming that a two-factor solution provided the best description of the data according to parallel analysis, BIC, and RMSEA.

We next assessed whether the measure could be shortened by removing items with the lowest loadings on each factor while retaining its internal consistency and factor structure. In addition to shortening the measure, the extremely high reliability of our scale suggested that there was some redundancy in the items, and as a result the investigation of a shortened version was warranted. We removed items with the weakest loadings until we were left with four items with primary loadings on each factor. In this reduced dataset, reliability was slightly reduced but still high (alpha $=.91$, omega $=.94$ ). Repeating the EFA again revealed the presence of two factors. 
Table 1. Factor loadings for initial two-factor exploratory factor analysis model. Loadings below .2 are hidden for ease of interpretation. Items retained for confirmatory factor analyses are highlighted in bold.

\begin{tabular}{lcc} 
Item & Factor 1 & Factor 2 \\
\hline 1. Touching surfaces & 0.17 & 0.62 \\
2. Close contact with stranger & 0.44 & 0.40 \\
3. Leaving residence and seeing others & 0.77 & -0.10 \\
4. Going to school or work & 0.77 & -0.10 \\
5. Going to grocery store & 0.85 & 0.00 \\
6. Avoiding contact with strangers & 0.67 & 0.10 \\
7. Touching face & 0.14 & 0.60 \\
9. Hearing someone cough & -0.03 & 0.92 \\
11. Coming within 6ft of strangers & 0.68 & 0.19 \\
13. Speaking to someone within 6ft & 0.75 & 0.11 \\
14. Store staff not wearing masks & 0.76 & -0.01 \\
17. Entering elevator with others & 0.57 & 0.23 \\
20. Coughing in my direction & 0.00 & 0.85 \\
22. Leaving home & 0.78 & -0.12 \\
27. Taking a taxi & 0.49 & 0.28
\end{tabular}

\section{Study 2}

Data quality and reliability

We first removed 9 subjects who did not pass the attention check, before testing reliability in this second sample. The measure again demonstrated high reliability (alpha $=.89$, omega $=.91$ ).

\section{Confirmatory factor analysis}

We next performed confirmatory factor analysis using the model structure determined using EFA in Study 1 , with four items loading on to each of the two factors. As we observed in Study 1 that the two factors were highly correlated, we also tested a single factor model in which all eight items loaded on to a single virus infection fear factor.

Fit statistics indicated that while the one factor model performed significantly worse than the two-factor model $\left(\Delta X^{2}=54.76, p<.001\right)$, the two-factor model did not provide a universally excellent fit to the data (Table 2). While the majority of fit statistics were in the good-to-excellent range, the RMSEA for the twofactor model was .10 and the $X^{2}$ was highly significant $(p<.001)$. As a result, we inspected the model modification indices to identify potential problems in the model specification. This revealed high covariance between two items ( 9 and 20), and so we re-ran the CFA incorporating a term representing correlated error variance between these items. This substantially and significantly improved model fit for both the one-factor $\left(\Delta X^{2}=116.84, p<.001\right)$ and two-factor $\left(\Delta X^{2}=67.77, p<.001\right)$ models, with all fit statistics now in the excellent range (Table 2 ). 
Avoidance of Respiratory Viral Infection Scale

Table 2. Model fit indices for confirmatory factor analysis models

\begin{tabular}{|c|c|c|c|c|c|c|c|c|}
\hline Model & $x^{2}$ & $d f$ & $p$ & RMSEA & $\mathrm{CFI}$ & SRMR & AIC & $\mathrm{BIC}$ \\
\hline \multicolumn{9}{|l|}{ 8-item } \\
\hline $\begin{array}{l}\text { Two factor, } \\
\text { covariance term }\end{array}$ & 36.359 & 18 & 0.006 & 0.055 & 0.985 & 0.028 & 7147.27 & 7216.25 \\
\hline $\begin{array}{l}\text { One factor, } \\
\text { covariance term }\end{array}$ & 55.769 & 19 & $<.001$ & 0.075 & 0.971 & 0.035 & 7164.68 & 7229.828 \\
\hline Two factor & 83.845 & 19 & $<.001$ & 0.1 & 0.948 & 0.048 & 7192.76 & 7257.905 \\
\hline One factor & 138.605 & 20 & $<.001$ & 0.132 & 0.905 & 0.051 & 7245.52 & 7306.833 \\
\hline \multicolumn{9}{|l|}{ 7-item } \\
\hline One factor & 23.152 & 9 & 0.006 & 0.068 & 0.981 & 0.027 & 5603.40 & 5649.391 \\
\hline Two factor & 47.973 & 14 & $<.001$ & 0.084 & 0.964 & 0.036 & 6479.83 & 6533.482 \\
\hline
\end{tabular}

Table 3. Standardized loadings from confirmatory factor analysis for 7 item scales with one and two factor solutions.

Item

Two factor model

1. Touching surfaces

5. Going to grocery store

7. Touching face

9. Hearing someone cough

13. Speaking to someone within $6 \mathrm{ft}$

14. Store staff not wearing masks

22. Leaving home

One factor model

1. Touching surfaces

5. Going to grocery store

7. Touching face

9. Hearing someone cough

13. Speaking to someone within $6 \mathrm{ft}$

14. Store staff not wearing masks

22. Leaving home
Factor 1

Factor 2

0.73

0.82

0.70

0.70

$\begin{array}{ll}- & 0.82\end{array}$

$\begin{array}{ll}- & 0.71\end{array}$

$\begin{array}{ll}- & 0.68\end{array}$

0.67

0.71

0.66

0.67

0.80

0.70

0.68 
However, the necessity of this correlated error covariance term makes the interpretation of the model unclear, and such correlated error terms can be problematic if not included for clear theoretical reasons (Hermida, 2015; Landis et al., 2009). As such, it was desirable to find a simpler solution to account for, or remove, this covariance. To achieve this, we elected to remove the item in this pair with the lower factor loading (20) and repeat the CFA. After removing this item, both one and two-factor solutions demonstrated an excellent fit to the data, with the two-factor solution providing a significantly better fit than the one-factor model $\left(\Delta X^{2}=18.95, p<.001\right)$. However, while the two-factor model provided the best model fit, inspecting the parameter estimates from the model indicated a very high correlation between the two factors $(r=.88)$. Correlations this high are regarded as problematic, as they indicate that the separate factors are likely to have poor discriminant validity (Brown, 2015). In this situation, it is desirable to seek a more parsimonious solution, assuming it still provides similar model fit. We therefore determined that the one-factor solution provided the best model in this case, given its good-to-excellent fit and parsimony. Our final measure therefore included seven items and had a unidimensional structure (Table 3 \& 4).

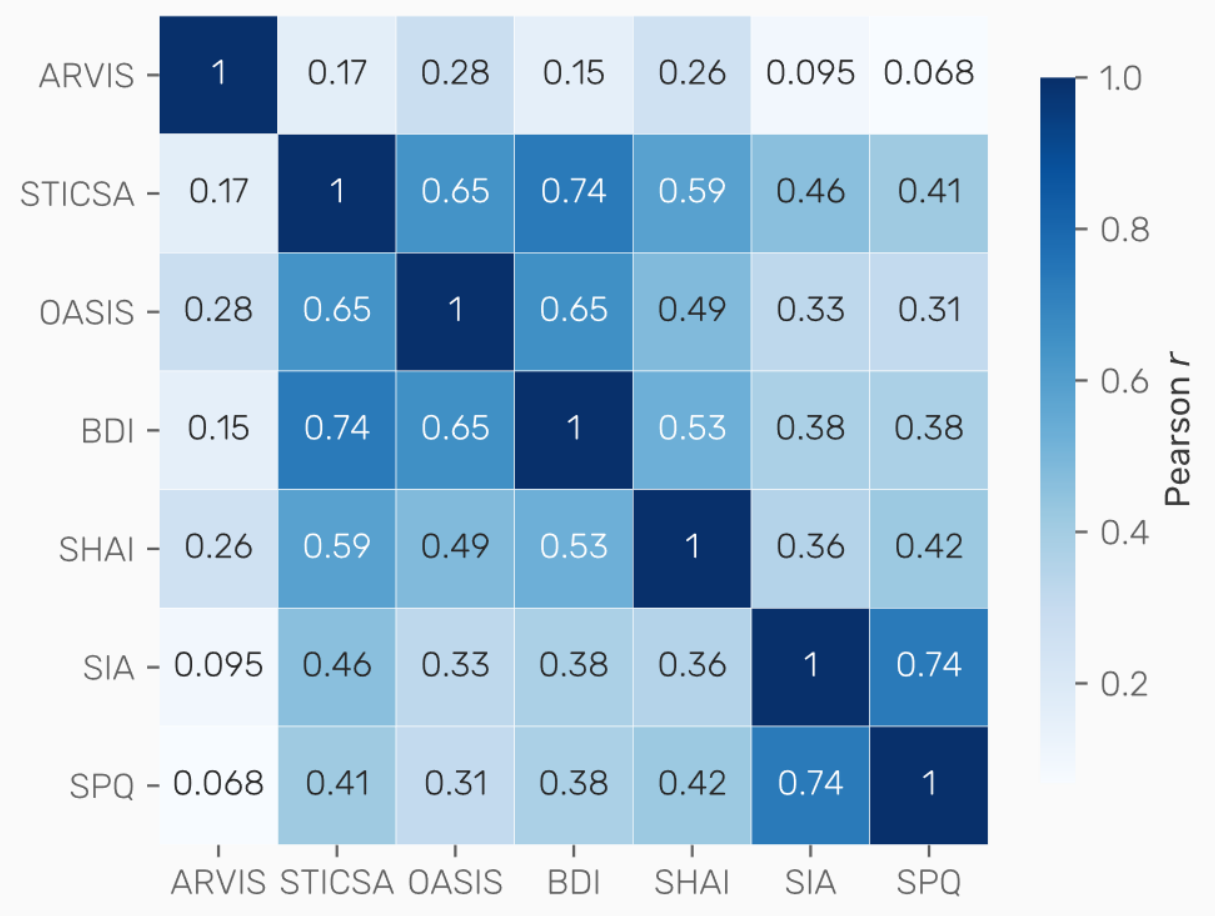

Figure 4. Correlations between the ARVIS and other measures chosen to assess convergent and discriminant validity in the sample from Study 2. The COVID threat scale was measured in a different sample (Study 1) and is therefore not shown here. ARVIS: Avoidance of Respiratory Viral Infection Scale, STICSA: State-Trait Inventory of Cognitive and Somatic Anxiety, OASIS: Overall Anxiety Severity and Impairment Scale, BDI: Beck Depression Inventory, SHAI: Short Health Anxiety Inventory, SIA: Social Interaction Anxiety, SPQ: Social Phobia Questionnaire.

\section{Convergent and discriminant validity}

Correlations between measures are shown in Figure 4, and overall demonstrate low correlations between the ARVIS and other measures, although most were significant in this sample. This is not unexpected, given the nature of the ARVIS as a state, partially environmentally dependent measure that theoretically should not be highly related with trait measures. Importantly, the ARVIS had the highest correlations with the OASIS, representing state anxiety $(r=.28, p<.001)$, although even this was modest. It was also modestly 
correlated with the SHAI, measuring general health anxiety $(r=.26, p<.001)$. Importantly, it was not significantly correlated with either social anxiety scales (SIAS $r=.10, p=.12$; SPS $r=.07, p=.26$ ), indicating that it is not simply measuring social anxiety traits.

The critical test of convergent and divergent validity, however, is the test of the difference between measures expected to be correlated and those expected to be uncorrelated (or weakly correlated). We therefore tested the significance of the difference in correlations between the OASIS and SHAI and all other measures using the method developed by Hittner et al. (2003), with the aim of determining whether the ARVIS correlated with the OASIS and SHAI to a greater extent than with other measures. This indicated that correlations between the ARVIS and OASIS were higher than correlations between the ARVIS and all other measures (excluding the SHAI), as shown in Table 5. Correlations between the ARVIS and SHAI were significantly higher than those with the SIAS and SPS, however they were not significantly higher than those between the ARVIS and BDI $(p=.07)$ or STICSA $(p=.08)$.

We additionally collected responses on a validated COVID threat scale (Kachanoff et al., 2020) in Study 1. ARVIS scores in this sample were modestly positively correlated with the realistic threat subscale of this measure, representing health and financial effects $(r=.27, p<.001)$. In contrast, ARVIS scores were negatively correlated, albeit to a lesser extent, with the symbolic threat subscale, representing threat to American values ( $r=-.20, p=.002)$. The difference between these correlations was significant (Table 5$)$.

Criterion validity

Finally, we examined whether the ARVIS was associated with reported real-world behavior with which we expected it to correlate, as individuals who are more fearful of everyday situations should be more likely to engage in behaviors associated with infection risk. This demonstrated that ARVIS scores were significantly correlated with reported avoidance of social interactions $(\rho=.43, p<.001$, Spearman correlations used due to non-normality), handwashing $(\rho=.29, p<.001)$, and staying home $(\rho=.27, p<.001)$.

Table 5. Comparisons between correlations with convergent and divergent validity measures. Z represents the strength of the difference between correlation coefficients.

\begin{tabular}{llrr} 
Convergent measure & Divergent measure & $\boldsymbol{Z}$ & $\boldsymbol{p}$ \\
\hline OASIS & STICSA & 2.34 & 0.02 \\
& BDI & 2.60 & 0.009 \\
& SIA & 2.72 & 0.007 \\
& SPQ & 3.07 & 0.002 \\
SHAI & STICSA & 1.75 & 0.08 \\
& BDI & 1.83 & 0.07 \\
& SIA & 2.44 & 0.02 \\
& SPQ & 2.99 & 0.003 \\
COVID realistic threat & COVID symbolic threat & 5.81 & $<.001$
\end{tabular}

\section{Study 3}

Internal consistency

The internal consistency in the data from the second time point was high (alpha $=.9$, omega $=.93$ ). 


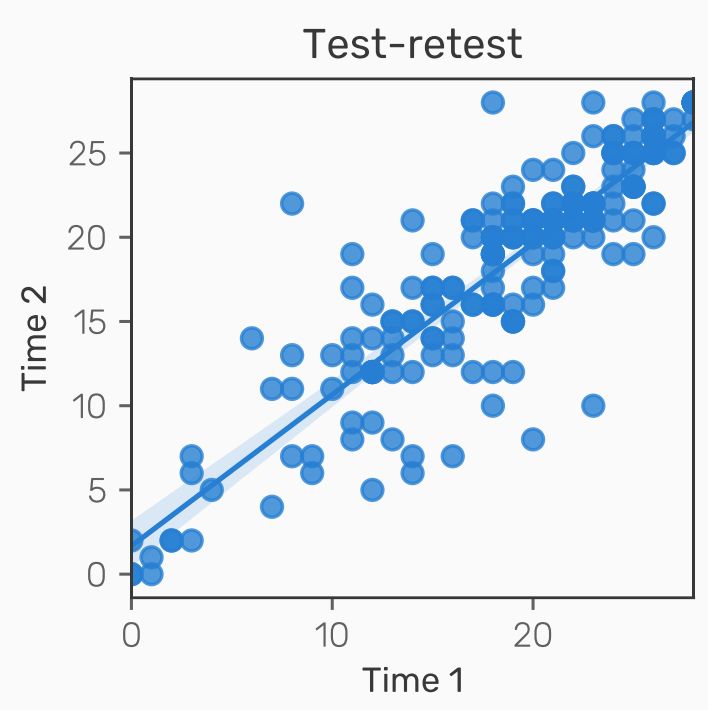

Figure 4. Correlation between scores on the ARVIS at time 1 and time 2

Test retest reliability

Test-retest reliability using Pearson correlations between scores at the two time points indicated that scores were strongly correlated $(r(200)=.88, p<.001)$. ICCs across the two time points showed that reliability was good according to both the ICC $(A, 1)(F(201,202)=16.4, p<.001)$ and the ICC $(C, 1)$ $(F(201,201)=16.4, p<.001)$.

\section{Discussion}

We aimed to develop and validate a questionnaire measure to assess fear of everyday situations and behaviors in the context of a respiratory virus pandemic. The resulting measure is short, unidimensional, shows good convergent and divergent validity, and predicts self-reported real-world behavior. While other measures have focused on explicit fear of viral infection, to our knowledge, this measure is the only one to explicitly measure fear of everyday situations in the context of a pandemic.

Through iterative exploratory and confirmatory factor analysis, we determined that while there was evidence for a two-factor structure, the most parsimonious model was a one-factor structure due to high correlation between the two identified factors. This suggests that virus-related fear is largely unidimensional, which may be a result of how it emerges. It is possible that virus-related anxiety is likely to be largely externally influenced, for example through government and media providing information about sources of infection risk, and therefore the structure represents a common source of fear across different subdomains. As such, we cannot rule out the possibility that the a two-factor structure may be more fitting when global, pervasive, sources of fear are less present. While there is little work on pandemic-related fear, previous studies on AIDS-related fear have identified separable dimensions (Arrindell et al., 1989), representing fear of interactions with others and fear of infection (Harrell \& Wright, 1998). This is reminiscent of the two-factor structure tested here where factors largely represented fear of encountering others and fear of infection. However, we found convincing evidence for a simpler, one factor solution. This discrepancy may be explained by the fact that this previous scale asked directly about fear of the virus itself, which was not the aim of the present study. 
In terms of psychometric measures, our questionnaire demonstrated high reliability, both in terms of internal consistency and of test-retest reliability over a one-week period according to multiple metrics. It also showed good validity according to multiple measures. When evaluating convergent and discriminant validity, we observed results that were largely in line with our expectations. The measure was most highly correlated with measures of state anxiety and health anxiety, realistic pandemic-related perceived threat, and less correlated with measures of depression, trait anxiety, and social anxiety. While none of these correlations were high, this is unsurprising given the highly state-dependent nature of the construct under evaluation and the fact that it is likely largely externally influenced, leaving relatively little room for relationships with trait measures. Interestingly, while we found our measure to correlate positively with a measure of realistic threat from COVID, we found a negative correlation with the symbolic threat subscale of this measure, indicating that individuals reporting that the pandemic posed a greater threat to national values were less fearful of everyday situations that pose an infection risk. Additionally, the positive correlation with realistic threat was modest, likely due to the distinction between perceived threat from the virus generally and specific fears of everyday situations. Of particular importance, we found no correlation between the ARVIS and social anxiety measures, indicating that items regarding avoidance of social interaction are not measuring social anxiety rather than virus-related fear of social encounters.

We also showed that the ARVIS measure has good criterion validity, demonstrating high correlations with self-reported engagement in protective behaviors such as social distancing and handwashing. This indicates that our measure concurrently predicts behaviors that we expect would be related to the construct it measures; individuals who are more fearful of social interaction for example should be more likely to avoid it. Critically, the measure did not assess engagement in these behaviors itself, only fear of the behavior, meaning that the correlation between ARVIS and reported protective behaviors are not due to overlap in the items used.

An important aspect of this questionnaire is the focus on fear of situations and behaviors that are typically not feared in the absence of pandemic respiratory viruses. For example, ordinarily the majority of individuals would not be expected to report a fear of coming within $6 \mathrm{ft}$ ( 2 meters) of other people. We chose to focus on the emotional reaction to everyday situations rather than fear of the virus itself, or of potential infection, for multiple reasons. First, this fear of everyday situations (associated with the virus) has the potential to be distressing and impairing in the absence of a pandemic. For example, while fear of viral infection itself is not necessarily pathological or impairing, fear of interacting with others due to perceived risk of viral infection could become distressing or impairing when it is no longer adaptive. Second, this fear may adaptively alter health behaviors if manipulated, for example by public health campaigns. While informing the public about the severity of the illness resulting from the virus itself may lead to fear of the virus, cultivating an adaptive level of fear relating to situations that can aid in viral spread, such as close social interaction, may help promote protective behaviors.

Finally, in the absence of a pandemic while many people would be fearful of a virus that has the potential to cause severe illness, the majority of people would not be fearful of the everyday situations targeted here. As a result, this fear represents a context-dependent learned fear of a previously safe stimulus, which will need to be modulated after the threat from the pandemic ceases to exist. From a learning theory perspective, this pandemic fits a positive occasion setting framework (Fraser \& Holland, 2019; Trask et al., 2017), where the specific stimulus or situation (e.g., social interactions within $6 \mathrm{ft}$ of each other) is not dangerous in most situations but is dangerous within the contextual positive occasion setter of a pandemic. Alternatively, this could be explained by a conditional stimulus pre-exposure effect (i.e., latent inhibition; (Lubow et al., 1981)). From this perspective, people did not pay attention to the threat/safety levels of these everyday situations with regards to contamination potential, but with the onset of the pandemic, this led to simple acquisition of 
fear of these situations; in which case, individuals would likely need to undergo extinction in order to reduce fear after the pandemic (in which the post-pandemic context for extinction would be a negative occasion setter). Indeed, there may be individual differences in the degree to which either of these accounts occurs. Overall, a pandemic represents a real-world example of a context-dependent fear conditioning experiment and could provide a test of lab-based conditioning theory in the real world.

This questionnaire also may help diagnostic assessment, especially following an epidemic or pandemic. To meet diagnostic criteria, an individual must exhibit distress or impairment that is not expected in the context in which it occurs (American Psychiatric Association, 2013). In a pandemic, the context itself is atypical, where elevated contamination fear during a pandemic is adaptive. For instance, prior to a pandemic, an individual showing fear of touching surfaces may receive a diagnosis and be treated accordingly. However, during the pandemic, diagnosis of an individual with this anxiety may be more challenging. After a pandemic, however, if individuals maintain elevated fears of these everyday situations, this could provide evidence towards a clinical diagnosis. While we did not evaluate this questionnaire with clinical vs non-clinical populations, future studies could investigate the clinical utility of our questionnaire.

While we believe the evidence provided here indicates that our measure is robust and valid, it is not without limitations. In particular, since the measure was developed and validated during the course of the COVID19 pandemic (May 2020), we were unable to validate the measure in the absence of a pandemic, and we therefore cannot conclude that the factor structure will remain identical when general levels of virus-related fear are lower. Additionally, we focused on subjects in the United States, where media and government communications largely discussed social distancing and infection control measures as a route to reducing viral transmission during the COVID-19 pandemic. It is possible that a different structure may emerge in environments where there is less clear information about these protective behaviors (e.g. the United Kingdom). We also did not test our measure in a clinical sample, for example subjects with pathological anxiety, where responses may be qualitatively different. Finally, our scale was evaluated in an online sample, which may mean it does not translate to in-person administration, although online samples are typically more representative of the population than samples used in the lab (Berinsky et al., 2012).

In conclusion, we have developed and validated a measure to asses fear of everyday situations in the context of a respiratory virus pandemic. This measure can be useful in assessing the extent to which individuals are experiencing fear and anxiety in their everyday life in the context of respiratory viral pandemics, and evaluating how this fear develops over the course of a pandemic and beyond. 


\section{References}

American Psychiatric Association. (2013). Diagnostic and Statistical Manual of Mental Disorders (DSM-5). American Psychiatric Association.

Arpaci, I., Karataş, K., \& Baloğlu, M. (2020). The development and initial tests for the psychometric properties of the COVID-19 Phobia Scale (C19P-S). Personality and Individual Differences, 164, 110108. https://doi.org/10.1016/j.paid.2020.110108

Arrindell, W. A., Ross, M. W., Robert Bridges, K., van Hout, W., Hofman, A., \& Sanderman, R. (1989). Fear of aids: Are there replicable, invariant questionnaire dimensions? Advances in Behaviour Research and Therapy, 11(2), 69-115. https://doi.org/10.1016/0146-6402(89)90015-5

Beck, A. T. (1978). The Beck Depression Inventory. Psychological Corporation.

Berinsky, A. J., Huber, G. A., \& Lenz, G. S. (2012). Evaluating Online Labor Markets for Experimental Research: Amazon.com's Mechanical Turk. Political Analysis, 20(3), 351-368.

https://doi.org/10.1093/pan/mpr057

Bish, A., \& Michie, S. (2010). Demographic and attitudinal determinants of protective behaviours during a pandemic: A review. British Journal of Health Psychology, 15(4), 797-824.

https://doi.org/10.1348/135910710X485826

Breslau, N. (2009). The Epidemiology of Trauma, PTSD, and Other Posttrauma Disorders. Trauma, Violence, \& Abuse, 10(3), 198-210. https://doi.org/10.1177/1524838009334448

Bromet, E. J., Hobbs, M. J., Clouston, S. a. P., Gonzalez, A., Kotov, R., \& Luft, B. J. (2016). DSM-IV posttraumatic stress disorder among World Trade Center responders 11-13 years after the disaster of 11 September 2001 (9/11). Psychological Medicine, 46(4), 771-783.

https://doi.org/10.1017/S0033291715002184

Brown, T. A. (2015). Confirmatory Factor Analysis for Applied Research, Second Edition. Guilford Publications.

Browne, M. W., \& Cudeck, R. (1992). Alternative Ways of Assessing Model Fit. Sociological Methods \& Research, 21(2), 230-258. https://doi.org/10.1177/0049124192021002005

Bults, M., Beaujean, D. J., de Zwart, O., Kok, G., van Empelen, P., van Steenbergen, J. E., Richardus, J. H., \& Voeten, H. A. (2011). Perceived risk, anxiety, and behavioural responses of the general public during the 
Avoidance of Respiratory Viral Infection Scale

early phase of the Influenza A ( $\mathrm{H} 1 \mathrm{~N} 1)$ pandemic in the Netherlands: Results of three consecutive online surveys. BMC Public Health, 11(1), 2. https://doi.org/10.1186/1471-2458-11-2

Campbell-Sills, L., Norman, S. B., Craske, M. G., Sullivan, G., Lang, A. J., Chavira, D. A., Bystritsky, A., Sherbourne, C., Roy-Byrne, P., \& Stein, M. B. (2009). Validation of a Brief Measure of Anxiety-Related Severity and Impairment: The Overall Anxiety Severity and Impairment Scale (OASIS). Journal of Affective Disorders, 112(1-3), 92-101. https://doi.org/10.1016/j.jad.2008.03.014

Clark, L. A., \& Watson, D. (2019). Constructing validity: New developments in creating objective measuring instruments. Psychological Assessment, 31(12), 1412-1427. https://doi.org/10.1037/pas0000626

Conway, L. G., Woodard, S. R., \& Zubrod, A. (2020). Social Psychological Measurements of COVID-19: Coronavirus Perceived Threat, Government Response, Impacts, and Experiences Questionnaires [Preprint]. PsyArXiv. https://doi.org/10.31234/osf.io/z2×9a

Cowling, B. J., Ip, D. K. M., Fang, V. J., Suntarattiwong, P., Olsen, S. J., Levy, J., Uyeki, T. M., Leung, G. M., Malik Peiris, J. S., Chotpitayasunondh, T., Nishiura, H., \& Mark Simmerman, J. (2013). Aerosol transmission is an important mode of influenza A virus spread. Nature Communications, 4(1), 1935.

https://doi.org/10.1038/ncomms2922

de Winter, J. C. F., Dodou*, D., \& Wieringa, P. A. (2009). Exploratory Factor Analysis With Small Sample Sizes. Multivariate Behavioral Research, 44(2), 147-181. https://doi.org/10.1080/00273170902794206

Dunn, T. J., Baguley, T., \& Brunsden, V. (2014). From alpha to omega: A practical solution to the pervasive problem of internal consistency estimation. British Journal of Psychology, 105(3), 399-412.

https://doi.org/10.1111/bjop.12046

Dutheil, F., Mondillon, L., \& Navel, V. (2020). PTSD as the second tsunami of the SARS-Cov-2 pandemic. Psychological Medicine, 1-2. https://doi.org/10.1017/S0033291720001336

Dziuban, C. D., \& Shirkey, E. C. (1974). When is a correlation matrix appropriate for factor analysis? Some decision rules. Psychological Bulletin, 81(6), 358-361. https://doi.org/10.1037/h0036316

Fergus, T. A., Valentiner, D. P., Kim, H.-S., \& McGrath, P. B. (2014). The Social Interaction Anxiety Scale (SIAS) and the Social Phobia Scale (SPS): A comparison of two short-form versions. Psychological Assessment, 26(4), 1281-1291. https://doi.org/10.1037/a0037313 
Fraser, K. M., \& Holland, P. C. (2019). Occasion setting. Behavioral Neuroscience, 133(2), 145-175. https://doi.org/10.1037/bne0000306

Grös, D. F., Antony, M. M., Simms, L. J., \& McCabe, R. E. (2007). Psychometric properties of the State-Trait Inventory for Cognitive and Somatic Anxiety (STICSA): Comparison to the State-Trait Anxiety Inventory (STAI). Psychological Assessment, 19(4), 369-381. https://doi.org/10.1037/1040-3590.19.4.369

Harper, C. A., Satchell, L. P., Fido, D., \& Latzman, R. D. (2020). Functional Fear Predicts Public Health Compliance in the COVID-19 Pandemic. International Journal of Mental Health and Addiction.

https://doi.org/10.1007/s11469-020-00281-5

Harrell, J. P., \& Wright, L. W. (1998). The Development and Validation of the Multicomponent AIDS Phobia Scale. Journal of Psychopathology and Behavioral Assessment, 20(3), 201-216.

https://doi.org/10.1023/A:1023020617248

Hayton, J. C., Allen, D. G., \& Scarpello, V. (2004). Factor Retention Decisions in Exploratory Factor Analysis: A Tutorial on Parallel Analysis. Organizational Research Methods, 7(2), 191-205.

https://doi.org/10.1177/1094428104263675

Hermida, R. (2015). The problem of allowing correlated errors in structural equation modeling: Concerns and considerations. Computational Methods in Social Sciences, 3(1), 5-17.

Hittner, J. B., May, K., \& Silver, N. C. (2003). A Monte Carlo evaluation of tests for comparing dependent correlations. The Journal of General Psychology, 130(2), 149-168.

https://doi.org/10.1080/00221300309601282

Kachanoff, F., Bigman, Y., Kapsaskis, K., \& Gray, K. (2020). Measuring Realistic and Symbolic Threats of COVID-19 and their Unique Impacts on Wellbeing and Adherence to Public Health Behaviors [Preprint]. PsyArXiv. https://doi.org/10.31234/osf.io/5zr3w

Killingley, B., \& Nguyen-Van-Tam, J. (2013). Routes of influenza transmission. Influenza and Other Respiratory Viruses, 7(s2), 42-51. https://doi.org/10.1111/irv.12080

Koo, T. K., \& Li, M. Y. (2016). A Guideline of Selecting and Reporting Intraclass Correlation Coefficients for Reliability Research. Journal of Chiropractic Medicine, 15(2), 155-163.

https://doi.org/10.1016/j.jcm.2016.02.012 
Avoidance of Respiratory Viral Infection Scale

Lai, C.-C., Shih, T.-P., Ko, W.-C., Tang, H.-J., \& Hsueh, P.-R. (2020). Severe acute respiratory syndrome coronavirus 2 (SARS-CoV-2) and coronavirus disease-2019 (COVID-19): The epidemic and the challenges. International Journal of Antimicrobial Agents, 55(3), 105924.

https://doi.org/10.1016/j.ijantimicag.2020.105924

Landis, R., Edwards, B., \& Cortina, J. (2009). Correlated residuals among items in the estimation of measurement models. Statistical and Methodological Myths and Urban Legends: Doctrine, Verity, and Fable in the Organizational and Social Sciences, 195-214.

Leung, N. H. L., Chu, D. K. W., Shiu, E. Y. C., Chan, K.-H., McDevitt, J. J., Hau, B. J. P., Yen, H.-L., Li, Y., Ip, D. K. M., Peiris, J. S. M., Seto, W.-H., Leung, G. M., Milton, D. K., \& Cowling, B. J. (2020). Respiratory virus shedding in exhaled breath and efficacy of face masks. Nature Medicine, 26(5), 676-680. https://doi.org/10.1038/s41591-020-0843-2

Lubow, R. E., Weiner, I., \& Schnur, P. (1981). Conditioned Attention Theory. In G. H. Bower (Ed.), Psychology of Learning and Motivation (Vol. 15, pp. 1-49). Academic Press.

https://doi.org/10.1016/S0079-7421(08)60171-1

Madhav, N., Oppenheim, B., Gallivan, M., Mulembakani, P., Rubin, E., \& Wolfe, N. (2017). Pandemics: Risks, Impacts, and Mitigation. In D. T. Jamison, H. Gelband, S. Horton, P. Jha, R. Laxminarayan, C. N. Mock, \& R. Nugent (Eds.), Disease Control Priorities: Improving Health and Reducing Poverty (3rd ed.). The International Bank for Reconstruction and Development / The World Bank. http://www.ncbi.nlm.nih.gov/books/NBK525302/

Maren, S., Phan, K. L., \& Liberzon, I. (2013). The contextual brain: Implications for fear conditioning, extinction and psychopathology. Nature Reviews Neuroscience, 14(6), 417-428. https://doi.org/10.1038/nrn3492

McGraw, K. O., \& Wong, S. P. (1996). Forming inferences about some intraclass correlation coefficients. Psychological Methods, 1(1), 30-46. https://doi.org/10.1037/1082-989X.1.1.30

Oxford, J. S. (2000). Influenza A pandemics of the 20th century with special reference to 1918: Virology, pathology and epidemiology. Reviews in Medical Virology, 10(2), 119-133. https://doi.org/10.1002/(SICI) 1099-1654(200003/04)10:2<119::AID-RMV272>3.0.CO;2-O 
Palan, S., \& Schitter, C. (2018). Prolific.ac_A subject pool for online experiments. Journal of Behavioral and Experimental Finance, 17, 22-27. https://doi.org/10.1016/j.jbef.2017.12.004

Perrin, P. C., McCabe, O. L., Everly, G. S., \& Links, J. M. (2009). Preparing for an Influenza Pandemic: Mental Health Considerations. Prehospital and Disaster Medicine, 24(3), 223-230.

https://doi.org/10.1017/S1049023X00006853

Perry, J. L., Nicholls, A. R., Clough, P. J., \& Crust, L. (2015). Assessing Model Fit: Caveats and Recommendations for Confirmatory Factor Analysis and Exploratory Structural Equation Modeling. Measurement in Physical Education and Exercise Science, 19(1), 12-21.

https://doi.org/10.1080/1091367X.2014.952370

Rosseel, Y. (2012). lavaan: An R Package for Structural Equation Modeling. Journal of Statistical Software, 048(i02). https://ideas.repec.org/a/jss/jstsof/v048i02.html

Salkovskis, P. M., Rimes, K. A., Warwick, H. M. C., \& Clark, D. M. (2002). The Health Anxiety Inventory: Development and validation of scales for the measurement of health anxiety and hypochondriasis. Psychological Medicine, 32(5), 843-853. https://doi.org/10.1017/S0033291702005822

Spielberger, C. D. (1983). Manual for the State-Trait Anxiety Inventory STAI (Form Y) ("Self-Evaluation Questionnaire"). http://ubir.buffalo.edu/xmlui/handle/10477/1873

Tellier, R. (2009). Aerosol transmission of influenza A virus: A review of new studies. Journal of The Royal Society Interface, 6(suppl_6), S783-S790. https://doi.org/10.1098/rsif.2009.0302.focus

Trask, S., Thrailkill, E. A., \& Bouton, M. E. (2017). Occasion setting, inhibition, and the contextual control of extinction in Pavlovian and instrumental (operant) learning. Behavioural Processes, 137, 64-72. https://doi.org/10.1016/j.beproc.2016.10.003

Wheaton, M. G., Abramowitz, J. S., Berman, N. C., Fabricant, L. E., \& Olatunji, B. O. (2012). Psychological Predictors of Anxiety in Response to the H1N1 (Swine Flu) Pandemic. Cognitive Therapy and Research, 36(3), 210-218. https://doi.org/10.1007/s10608-011-9353-3

Wise, T., Zbozinek, T. D., Michelini, G., Hagan, C. C., \& mobbs, dean. (2020). Changes in risk perception and protective behavior during the first week of the COVID-19 pandemic in the United States [Preprint]. PsyArXiv. https://doi.org/10.31234/osf.io/dz428 
Wolf, E. J., Harrington, K. M., Clark, S. L., \& Miller, M. W. (2013). Sample Size Requirements for Structural Equation Models: An Evaluation of Power, Bias, and Solution Propriety. Educational and Psychological Measurement, 76(6), 913-934. https://doi.org/10.1177/0013164413495237

World Health Organisation. (2006). Nonpharmaceutical Interventions for Pandemic Influenza, National and Community Measures. Emerging Infectious Diseases, 12(1), 88-94.

https://doi.org/10.3201/eid1201.051371

Xu, J., Zheng, Y., Wang, M., Zhao, J., Zhan, Q., Fu, M., Wang, Q., Xiao, J., \& Cheng, Y. (2011). Predictors of symptoms of posttraumatic stress in Chinese university students during the $2009 \mathrm{H} 1 \mathrm{~N} 1$ influenza pandemic. Medical Science Monitor : International Medical Journal of Experimental and Clinical Research, 17(7), PH60-PH64. https://doi.org/10.12659/MSM.881836 


\section{Appendix}

During pandemics, many people become feel more anxious about doing things that they previously did every day. These questions ask how you feel about various everyday situations.

Answered on a 5-point scale: strongly disagree, moderately disagree, neither agree nor disagree, moderately agree, strongly agree

1. I am fearful of touching surfaces that others have touched

2. Going to a busy grocery store would make me anxious

3. I worry about touching my face after touching a surface other people have touched

4. I get worried if I hear someone cough near me

5. I would feel anxious if I had to speak to somebody standing less than $6 \mathrm{ft}$ from me

6. I would feel uncomfortable if I visited a store where the staff were not wearing masks

7. Leaving my home puts me in danger 


\section{Supplementary material}

Table S1. Initial items used for Study 1.

\begin{tabular}{cl} 
Question ID & Item \\
\hline 1 & I am fearful of touching surfaces that others have touched \\
2 & I feel anxious if I come into close contact with someone I don't know \\
3 & I am scared to leave my residence in case I come into contact with other people \\
5 & The thought of going to work or school makes me fearful \\
& Going to a busy grocery store would make me anxious \\
6 & A try to avoid situations where I might come into close contact with people I don't \\
7 & I worry about touching my face after touching a surface other people have touched \\
8 & I avoid touching door handles \\
9 & I get worried if I hear someone cough near me \\
10 & I wash my hands after touching surfaces that others have touched \\
11 & I feel uncomfortable if I come within 6ft of someone I don't know \\
12 & Attention check \\
13 & I would feel anxious if I had to speak to somebody standing less than 6ft from me \\
14 & I would feel uncomfortable if I visited a store where the staff were not wearing masks \\
15 & The thought of getting on a crowded bus or train makes me anxious \\
16 & I try to avoid touching people I don't know \\
17 & I would be scared to get in an elevator with other people \\
18 & I disinfect anything I bring into my house before using it \\
19 & I would feel less anxious if people wore masks in public places \\
20 & I would feel scared if someone coughed in my direction \\
21 & I try to keep at least $6 f t$ from people I don't know \\
22 & Leaving my home puts me in danger \\
23 & Getting on a busy plane would scare me \\
24 & The thought of going to a concert or sporting event with a crowd of people makes me \\
25 & anxious \\
26 & I would avoid going to a busy bar or restaurant \\
27 & I would be scared to take a taxi \\
&
\end{tabular}

